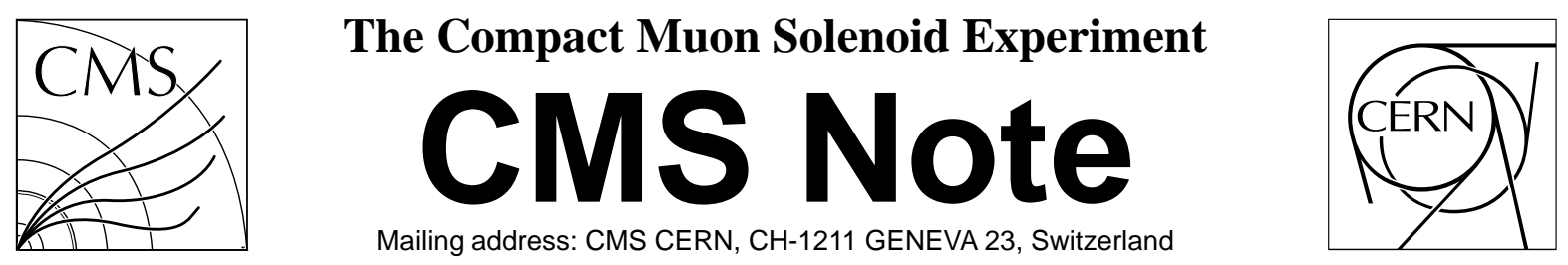

August 25, 1998

\title{
Spatial Resolution Attainable with Cathode Strip Chambers at the Trigger Level
}

\author{
M.M. Baarmand, A. Vaniachine ${ }^{1}$ \\ State University of New York at Stony Brook, NY 11790, USA \\ Yu. Bonushkin, C. Matthey, J. Hauser, T. Muller², S. Otwinowski, \\ J.Zeng \\ University of California, Los Angeles, CA 90024, USA \\ D. Chrisman, P. Giacomelli ${ }^{3}$, W. Gorn, J.G. Layter, C. Minor, P. Schenk ${ }^{4}$ \\ University of California, Riverside, CA 92521, USA \\ S. Durkin, J. Hoftiezer, P. Lennous, T.Y. Ling, C. Rush \\ Ohio State University, Columbus, OH 43210, USA \\ T. Ferguson, J. Hirschfelder, H. Hoorani \\ Carnegie Mellon University, Pittsburgh, PA 15213, USA \\ J. Fitch \\ Massachusetts Institute of Technology, Cambridge, MA 02139, USA \\ L. Gorn, A. Korytov, G. Mitselmakher \\ University of Florida, Gainesville, FL 32611, USA \\ O. Kisselev, O. Prokofiev, I. Smirnov, V. Soulimov \\ Petersburg Nuclear Physics Institute, Gatchina, Russia
}

1. Now at Royal Institute of Technology, Stockholm, Sweden.

2. Now at Institut fur Experimentelle Kernphysik, Karlsruhe, Germany

3. Now at INFN, Sezione di Bologna, Viale B.Pichat 6/2, Bologna, Italy.

4. Now at Andersen Consulting, Fredericton, New Brunswick, Canada. 


\title{
D.E. Klem, T. Vercelli, C. Wuest \\ Lawrence Livermore National Laboratory, Livermore, CA 94551, USA
}

\author{
S. Medved, L. Preston \\ Purdue University, West Lafayette, IN 47907, USA
}

\author{
J.C. Santiard \\ CERN, CH-1211, Geneva 23, Switzerland
}

\author{
E. von Goeler \\ Northeastern University, Boston, MA 02115, USA
}

\begin{abstract}
A simple network of comparators applied to the strip signals of a cathode strip chamber allows quick hit localization to within a halfstrip width, or +/- a quarter-strip. A six-plane chamber with $6.4 \mathrm{~mm}$ wide strips was tested in a high-energy muon beam. The chamber was placed behind a $30 \mathrm{~cm}$ thick iron block. We show that patterns of hits localized to within a halfstrip allowed us to identify $300 \mathrm{GeV} / \mathrm{c}$ muon tracks with $99 \%$ probability and $0.7 \mathrm{~mm}$ spatial resolution in the presence of bremsstrahlung radiation. This technique of finding muon tracks will be used in the cathode strip chambers of the CMS Endcap Muon System.
\end{abstract}

\section{Introduction}

The CMS Endcap Muon System is based on six-plane Cathode Strip Chambers (CSCs) placed between the $60 \mathrm{~cm}$ thick iron disks of the magnet return yolk [1]. To provide sharp turn-on curves for trigger thresholds up to $\mathrm{p}_{\mathrm{T}}=50-100 \mathrm{GeV} / \mathrm{c}$, the muon endcap chambers must be capable of identifying and measuring muon track stubs with a spatial precision of $1-2 \mathrm{~mm}$ in the bending plane at the first trigger level [2].

The technique described in this paper is based on a comparator network [3] which allows one to localize muon hits to within a halfstrip width (Fig. 1). If a strip has a signal amplitude which is above threshold and

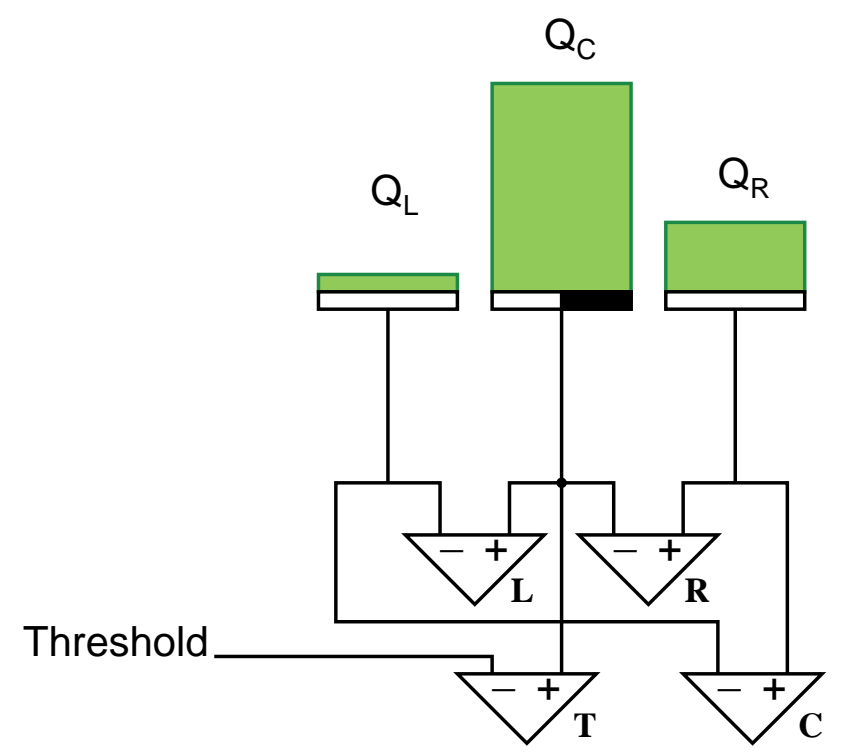

Fig. 1. The comparator network: four simultaneous comparisons of nearby charges allow one to localize a hit position in a cathode strip chamber within a halfstrip width. In this example, the results $\mathrm{L}+$, $\mathrm{R}-, \mathrm{C}+$ and $\mathrm{T}+$ select the halfstrip marked by the black rectangle. 
larger than those on the left and right neighboring strips, then this strip is identified as the local maximum in the cluster. In addition, by comparison of the relative amplitudes on the left and right neighbors, one can judge whether the muon passed through the left or right half of the central strip.

After identifying hits in all six planes to within halfstrip-wide intervals (see Fig. 2), one can look for pat-

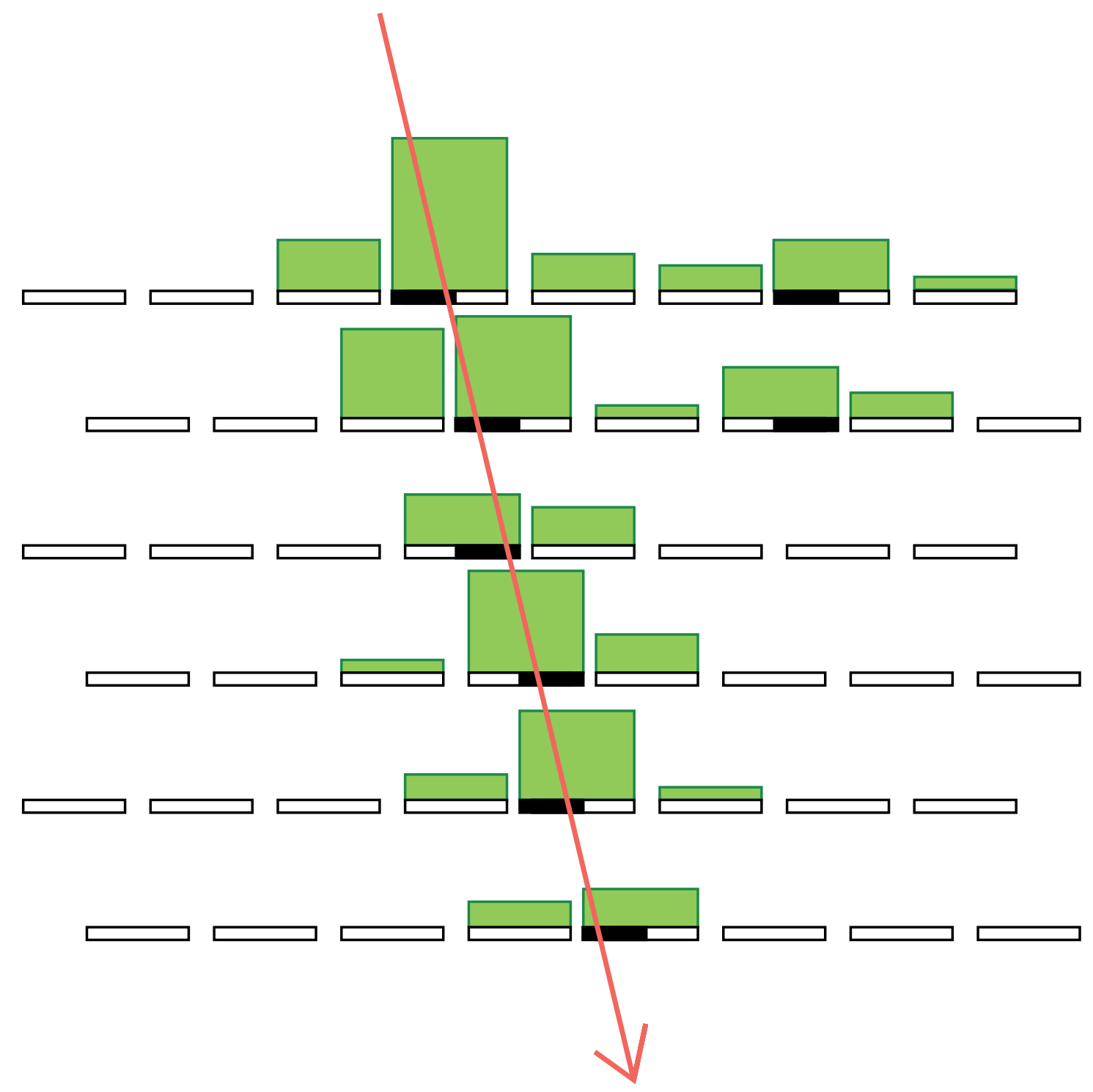

Fig. 2. Partial cross section of a CSC, showing schematically the six layers of strips (staggered by a halfstrip) and the charge induced on each strip. The pattern of hits in the six layers (black halfstripwide rectangles) can be compared with a list of valid track patterns to identify a muon. Each valid pattern has a very limited range of possible track coordinates and inclinations and, thus, one can obtain track coordinates with good spatial resolution online without any computation.

terns consistent with a muon track which satisfy certain selection criteria (e.g., range of inclination angles). This can be done by means of look-up tables when high speed is essential. A consistent pattern is called an LCT - a local charged track.

A cathode strip chamber prototype was thoroughly studied in a high-energy muon beam at the CERN laboratory. A set of data was taken with one out of six planes connected to a comparator network prototype board. The other five planes were read out by 10-bit ADCs; these planes were used to identify and fit each 
muon track and predict its position in the plane which had the comparator board. The first part of the paper describes these beam results and an analysis of different error contributions.

Detailed understanding of the board performance and error sources allowed us to implement them in a Monte Carlo simulation. By applying it to the real off-line data, we could simulate the expected trigger performance of the chamber if all its six planes were equipped with comparators. To do this analysis, we used a set of Si micro-strip detectors placed a few meters upstream of the chamber and in front of the iron slab so that we could obtain an unbiased prediction of the muon track position in the chamber. These results are described in the second part of the paper.

\section{Setup}

\subsection{Chamber and DAQ electronics}

A detailed description of the chamber design and DAQ electronics can be found elsewhere [4] - here we give a very short summary.

The chamber was made out of seven $16 \mathrm{~mm}$ thick panels forming six $9.5 \mathrm{~mm}$ gas gaps. Gold-plated tungsten 50 micron diameter wires were stretched with a spacing of $3.4 \mathrm{~mm}$ in the middle of these gaps. The sensitive area of the chamber was about $50 \times 50 \mathrm{~cm}^{2}$. Perpendicular to the wires, $6.35 \mathrm{~mm}$ wide parallel strips were milled on six panels (one strip plane per gas gap). The chamber was filled with an $\mathrm{Ar}+\mathrm{CO} 2+\mathrm{CF} 4=30 \%+40 \%+30 \%$ mixture and the nominal operating high voltage was $4.0 \mathrm{kV}$, giving about $100 \mathrm{fC}$ charge on a cathode plane per $300 \mathrm{GeV} / \mathrm{c}$ muon. The chamber had 32 active strips per plane.

Front-end cathode electronics for the standard DAQ path with charge digitization was based on the Gasplex chip [5]. With $500 \mathrm{~ns}$ shaping time and $160 \mathrm{pF}$ strip capacitance, the noise was measured to be $0.6 \mathrm{fC}$. The charges were digitized with 10-bit ADCs. The overall 10-bit range was $200 \mathrm{fC}$, beyond which the Gasplex quickly loses its linearity and goes into saturation.

\subsection{Halfstrip comparator network board}

A schematic of the preamplifier part of the comparator board is shown in Fig. 3. It was based on a onechannel version of the Gasplex chip. The overall amplification could be manually equalized between different channels at the second stage of amplification. The last RC pair was used to differentiate the pulse so that it peaked at $100 \mathrm{~ns}$ for a delta-function input charge. When convoluted with a real chamber signal, the output signal peaked at $150 \mathrm{~ns}$.

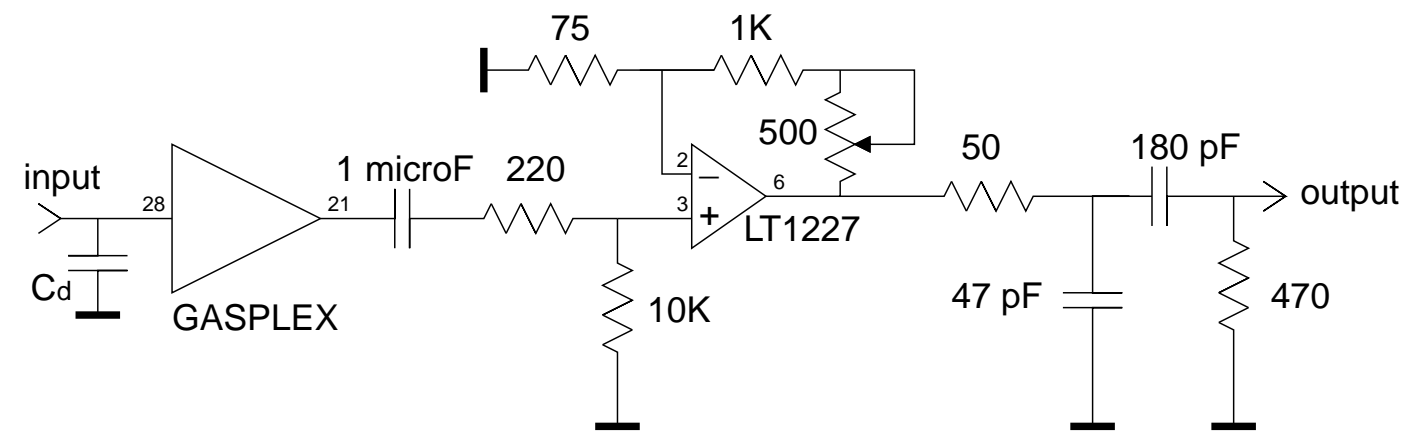

Fig. 3. Layout of the comparator board preamplifier based on the one-channel Gasplex chip. 
The comparator part of the board is shown in Fig. 4. Three comparators per channel were sufficient to have

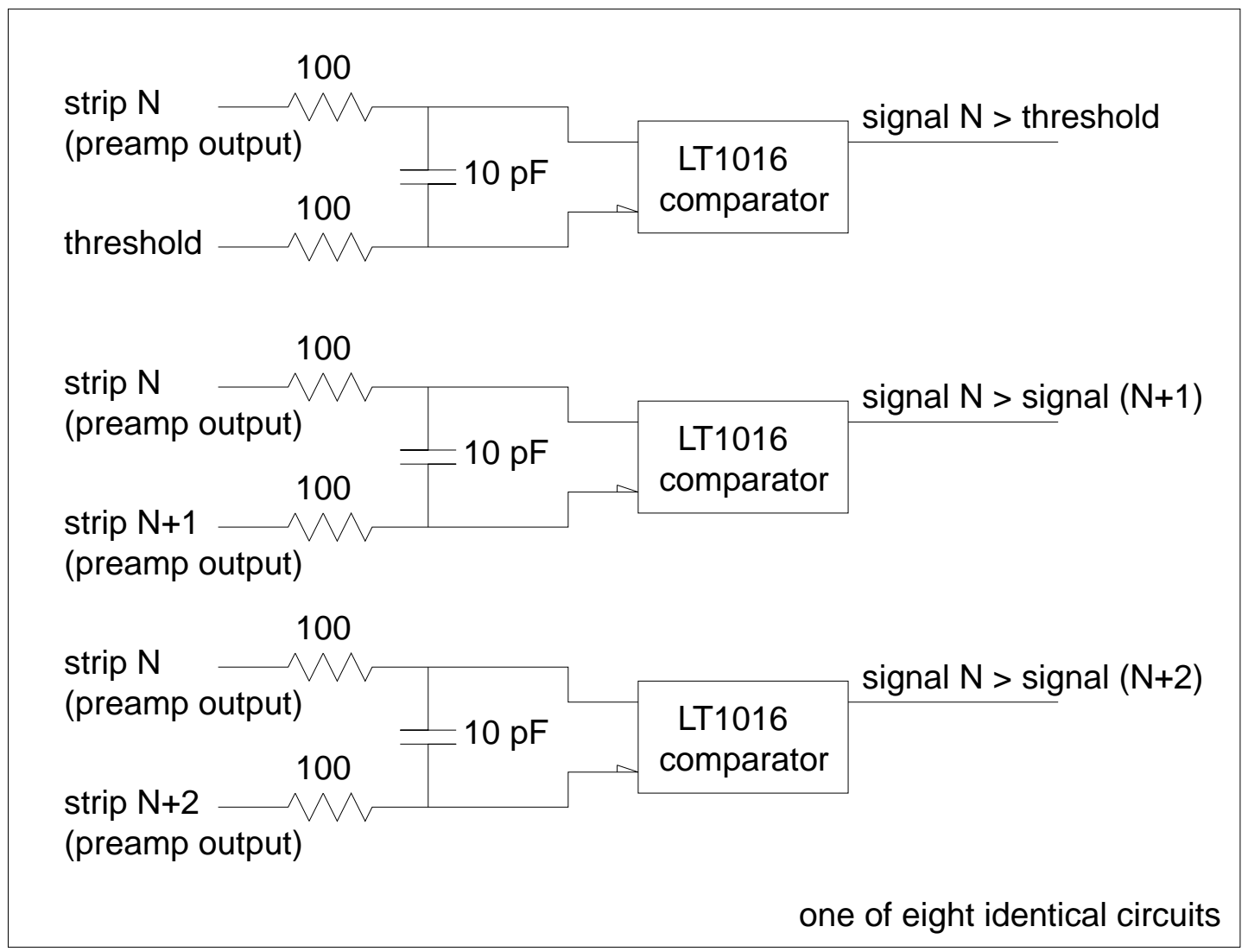

Fig. 4. Comparator network board: three comparators per channel provide all the necessary information to localize hits within a halfstrip wide interval. Comparators of adjacent strips are shared by adjacent channels.

all the necessary bits of information to localize a cluster within a halfstrip-wide interval. In the absence of a hit, the "signal-over-threshold" comparators were in a "no" state, while the others, by virtue of comparing two zeros, were randomly flip-flopping between "yes" and "no" states with a typical time between transitions of 20-80 ns. If a muon was detected, the state of all the comparators was latched in a LeCroy 4448 pattern register during a $20 \mathrm{~ns}$ gate. The gate was synchronized with the beam trigger and aligned with the time when the preamp output signals reached their maxima.

The noise of the entire system was measured by injecting a test pulse at the input and observing the relative probability of "yes" and "no" states of the signal-over-threshold comparators. We scanned signal amplitudes in the vicinity of the threshold and the resulting "probability vs. input signal" curve was fitted with the error function. This allowed us to obtain a direct measure of the noise - 1.1 fC (Gaussian sigma).

The prototype comparator board had 8 channels which were connected to strips 17 through 24 of the third chamber plane. Strips 25 through 32 were left floating, and strips 1 through 16 of this layer and all 32 strips in the other five layers were connected to the 16-channel Gasplex preamps and read out with ADCs.

\subsection{Experimental setup}

The setup for the beam test is shown in Fig. 5. The CSC was placed approximately perpendicular to the beam, with its strips vertical. The SiBT (Silicon Beam Telescope) five meters upstream of the CSC is a facility of the $\mathrm{H} 2$ beamline at CERN; it has a $2.6 \times 5.8 \mathrm{~cm}^{2}$ cross section and $7 \mu \mathrm{m}$ intrinsic resolution in both $x$ and $y$ projections, which translates to $100 \mu \mathrm{m}$ when extrapolated to the center of the CSC. 
For the comparator prototype tests, the beam was $100 \% 150 \mathrm{GeV} / \mathrm{c}$ muons at an angle $\alpha$ of $12.5 \mathrm{mrad}$ to the normal to the chamber, and with $\sigma(\alpha)=1.5 \mathrm{mrad}$. The beam was centered on strip 20 of layer 3 and data were taken with layer 3 at $3.9,4.0,4.1,4.2$ and $4.3 \mathrm{kV}$, corresponding to cathode charge distributions with Landau peaks at 60, 100, 170, 300 and $550 \mathrm{fC}$. The other five layers were kept at $4.0 \mathrm{kV}(100 \mathrm{fC})$.

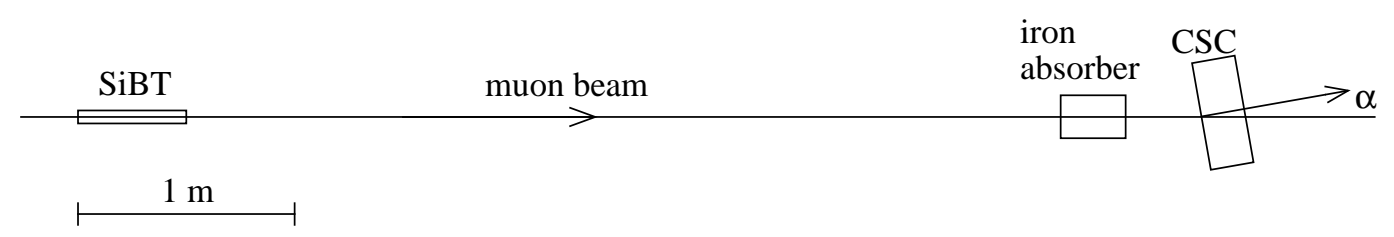

Fig. 5. Experimental setup in the $\mathrm{H} 2$ test beam, seen from above. The angle $\alpha$ between the CSC and the beamline is exaggerated for visibility. The iron absorber was only present for the second data set.

To study the LCT-finding performance, a second data set was taken with all strips connected to ADCs (without the comparator board) and with $30 \mathrm{~cm}$ of iron absorber placed approximately $25 \mathrm{~cm}$ upstream of the chamber. The iron simulates the magnet yokes which will be in front of the CSCs in the real CMS detector. The beam for this data was $100 \% 300 \mathrm{GeV} / \mathrm{c}$ muons with $\alpha=10.8 \mathrm{mrad}$ and $\sigma(\alpha)=1.2$ $\mathrm{mrad}$. The high voltage on all six layers was $4.0 \mathrm{kV}$.

\section{Single-layer results}

\subsection{Comparator prototype results}

The data from the comparators were used to measure the efficiency and accuracy of the peak halfstrip identification, and the incidence of extra peaks. Since the hardware prototype gave only comparator outputs, the logic for finding the peak halfstrip was implemented in software, starting from the pattern register data. The comparators gave enough information to find peaks in the 6 central strips (12 halfstrips).

The "correct" halfstrip was defined as the halfstrip in layer 3 which was crossed by the track fit to the hits in the other five layers. The resolution of a 5-layer trackfit in the CSC was $30 \mu \mathrm{m}(0.5 \%$ of the $6.35 \mathrm{~mm}$ stripwidth). An implication of this definition is that the efficiency results described in this section apply only to clusters associated with isolated tracks, because trackfits were only attempted for single-track events, defined as those with no more than one layer with multiple charge clusters. The question of trigger efficiency for more complicated topologies is addressed in the next section.

Figure 6 summarizes the results at $4.0 \mathrm{kV}$. Figures $6 \mathrm{a}$ and $6 \mathrm{~b}$ give an idea of the total rate and position accuracy of detected peaks. The main result is given in Fig. 6c, which shows the probability of finding the correct halfstrip vs. the track position. The local track position $x s$ is measured in stripwidths $(6.35 \mathrm{~mm})$ and defined to be zero at the center of a halfstrip, and \pm 0.25 at its edges. Figure $6 \mathrm{c}$ also shows the probability of finding either the correct halfstrip or the next closest halfstrip (dashed line). Integrating the probabilities over $x s$ gives a halfstrip efficiency of $92 \%$ and a one-strip efficiency of $98 \%$. To check for a difference in behavior at the center of strips and between strips, the same efficiencies are shown separately for left and right halfstrips in Figs. 6d and 6e. The right side of Fig. 6d and the left side of Fig. 6e correspond to strip centers, while the left side of Fig. $6 \mathrm{~d}$ and the right side of Fig. 6e are between strips; there is no significant difference. 

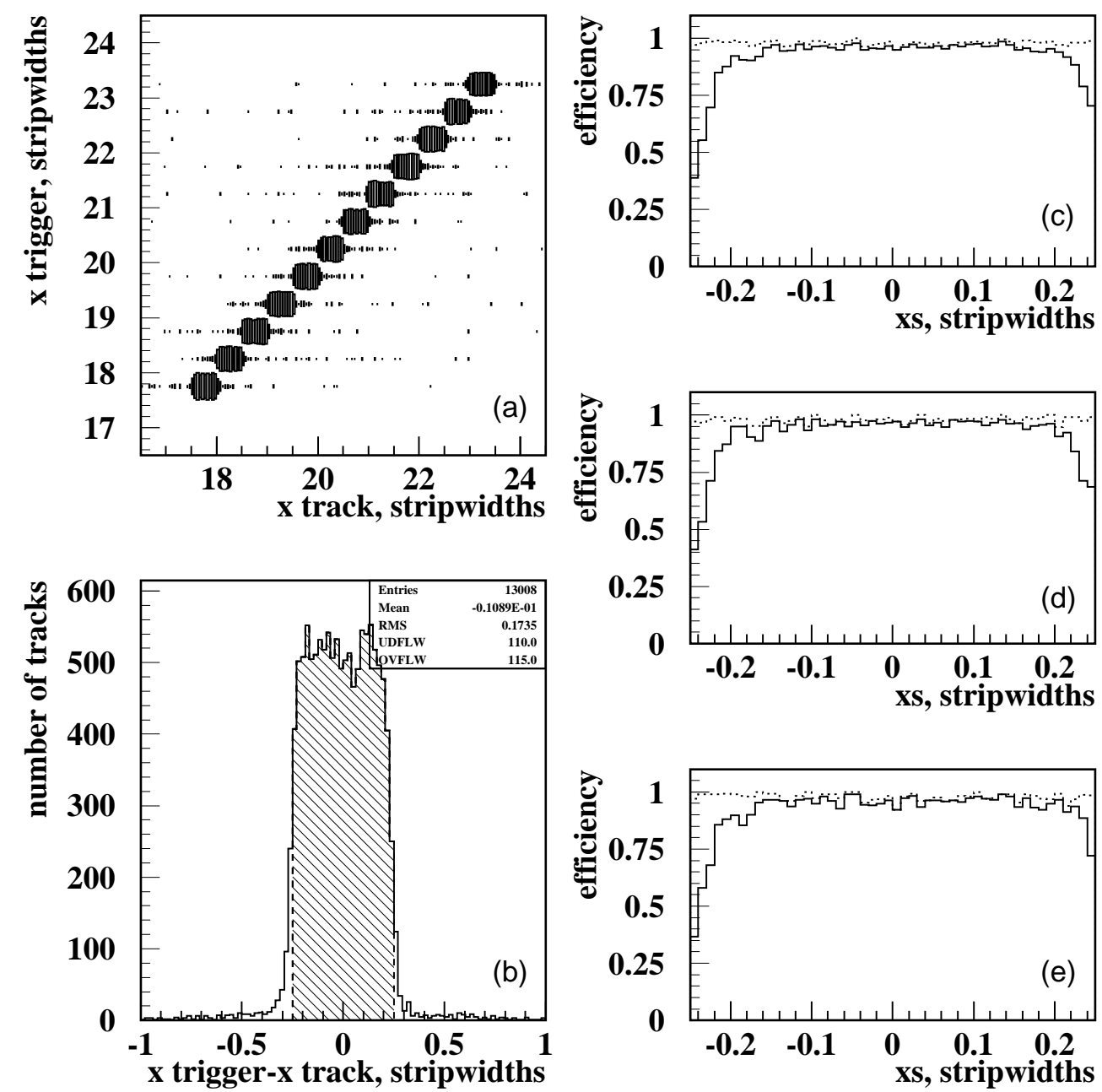

Fig. 6. Halfstrip comparator peakfinding performance at $4.0 \mathrm{kV}$, for isolated tracks: a) location of all halfstrip peaks vs. track position from a five-layer fit, b) distance to the track from the center of the nearest halfstrip peak, with the area corresponding to halfstrip resolution shaded, c) efficiency for finding the correct halfstrip (solid line) and either the correct or next-nearest halfstrip (dashed line), d) same efficiencies for left halfstrips, e) same for right halfstrips.

\subsection{Simulation of single-layer performance}

Two simulations have been developed. The first, called the "software trigger", simulates comparator results starting from strip ADC data. It was required to match several observed properties of the comparators: the halfstrip and one-strip efficiencies, the probabilities of the threshold and comparator bits being set, and the asymmetry at the halfstrip boundaries. The tuning needed to achieve this match provided an understanding of the comparator behavior, and also starting parameters for the second simulation, which is purely software and is referred to as "Monte Carlo". The Monte Carlo simulates both the CSC and the comparators and is used to study the dependence of the peakfinding efficiency on individual chamber and electronics parameters.

The trigger threshold could not be measured directly from the data because there was no ADC data for the 
strips connected to the comparators. So it was determined by looking at the strips in layer 3 crossed by tracks and adjusting the software threshold until the fraction of strips with the threshold bit set by software (strips 1-16) matched the fraction set by comparators (strips 17-24) at all five high voltages. A threshold of $15 \mathrm{fC}$ gave the best match and agreed well with the estimate of $15.7 \mathrm{fC}$ from the known reference voltage at the comparator input.

Figures $6 \mathrm{c}-\mathrm{e}$ show that most peakfinding errors are caused by a shift of the peak across a nearby halfstrip boundary to the neighbor halfstrip. Noise will cause a symmetric transfer across boundaries, while amplifier gain inequalities will cause an uneven transfer. A rough estimate of the gain uniformity was made by studying the pattern of asymmetries, halfstrip by halfstrip. The gain of each channel was also measured with test pulses. Table 1 shows the results of the two measurements, where the overall normalization of the first row has been adjusted to match the second row. Based on these results, the standard deviation of the gain was measured to be $3.5 \%$.

Tab. 1. Estimates of relative gain between strips in one layer from two different methods.

\begin{tabular}{l|rrrrrrrr}
\hline \multicolumn{1}{c|}{ strip } & 17 & 18 & 19 & 20 & 21 & 22 & 23 & 24 \\
\hline asymmetry & 1.04 & 1.02 & 1.00 & 0.96 & 0.98 & 1.00 & 0.98 & 1.02 \\
test pulsing & 1.10 & 1.02 & 1.00 & 0.95 & 0.97 & 0.99 & 0.96 & 1.02 \\
\hline
\end{tabular}

The probability in each event that a nearest-neighbor or next-nearest-neighbor comparator bit will be set is not 0.5 , but higher, because the pattern register latches the bit if the comparator switches on at any time during the gate. Each comparator also has an intrinsic input offset voltage. These two effects combined were measured by looking at the response of the comparators to noise. Events were selected which had tracks in a region far from the comparators, and the fraction of events for which each comparator bit was set was measured to be $0.59 \pm 0.01$. Lab measurements on some of the comparators confirmed that the fluctuations about the average value are roughly consistent with their intrinsic offsets. In the software trigger, only the average bias was simulated, by adding an offset of $0.5 \mathrm{fC}$ to each software comparator, which gave a comparator-on probability of 0.58 for all channels.

As mentioned in Sect. 2.2, the noise level was measured during bench tests of the comparators to be $1.1 \mathrm{fC}$ rms. An estimate of the noise when the comparators were installed on the chamber was made by using the software trigger, by varying the amount of noise added to each strip before simulating the comparator decisions, and looking at the efficiency near the halfstrip edges. As an example, Fig. 7 shows the simulated efficiency for three different noise levels, compared with the measured efficiency. This estimate gave $1.5 \pm 0.5 \mathrm{fC}$ for the added noise, or $1.6 \mathrm{fC}$ when added in quadrature to the $0.6 \mathrm{fC}$ ADC noise.

\subsection{Monte Carlo simulation}

A Monte Carlo simulation of the strip signals was used to look at the relative importance of various contributions to the trigger inefficiency. The Monte Carlo had already been developed and tuned to match the CSC ADC data quite well [4], so it could be extended to simulate the trigger performance simply by adding to it the same logic and parameters used for the software trigger. Summarizing the results above, these parameters were: trigger threshold $15 \mathrm{fC}$, trigger noise $1.62 \mathrm{fC}$, gain variation $3.5 \%$, and comparator bias $0.5 \mathrm{fC}$.

Figure 8 shows a comparison of the comparator halfstrip efficiency results from the prototype data and the two simulations as a function of the high voltage. The voltage-dependence is similar for all three, except at $4.3 \mathrm{kV}$, where a large percentage of clusters have strips with an ADC overflow (27\%). The ADCs saturate at $200 \mathrm{fC}$, while the comparator inputs become nonlinear at about $450 \mathrm{fC}$. The different saturation points are simulated in the Monte Carlo, but the software trigger is not able to recover the lost information from the ADC data. 


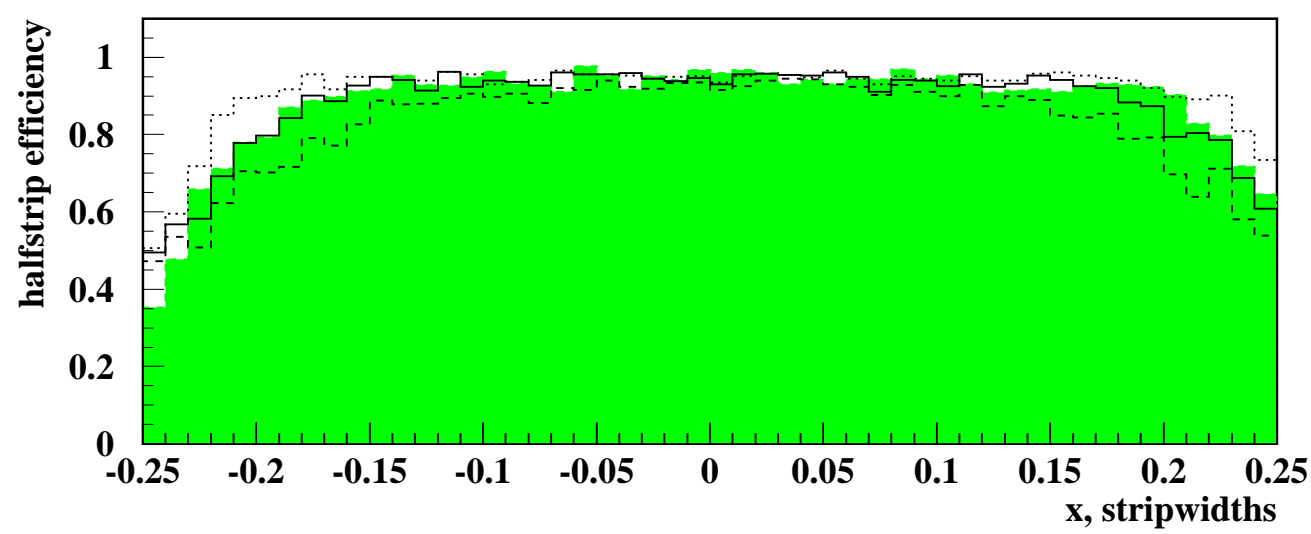

Fig. 7. The effect of noise on trigger efficiency near the halfstrip edges. The efficiency is shown as a function of the track position $x$ in the halfstrip for data (shaded) and for the software simulation with three values of added noise: $0 \mathrm{fC}$ (dotted line), $1.5 \mathrm{fC}$ (solid line) and $3.0 \mathrm{fC}$ (dashed line).

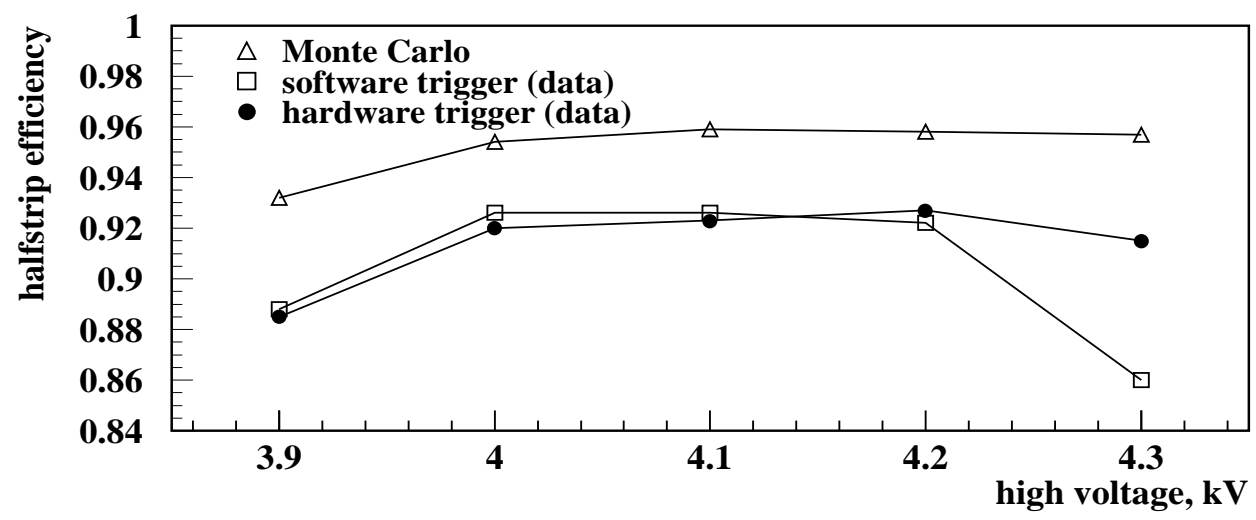

Fig. 8. Comparator halfstrip efficiency vs. high voltage: hardware prototype and two simulations.

The most important element missing from the Monte Carlo is any simulation of delta electrons, which are expected to occur at a rate of about 5\% per layer, and which appear in the data as broadened or multiple clusters. These delta rays are thought to be the main source of the constant $3.5 \%$ difference in efficiency between the Monte Carlo and the hardware and software triggers. They are also probably the explanation of the position-independent 2-3\% inefficiency seen in Figs 6c-e, an interpretation which is supported by visually scanning events in which a track passed near the center of a halfstrip.

Figure 9 shows the Monte Carlo halfstrip efficiency curve of Fig. 8, along with results for six cases where only one contribution to the trigger inefficiency has been turned on in the simulation. It shows that, in our prototype, the noise and gain inequalities were the most important sources of error. 


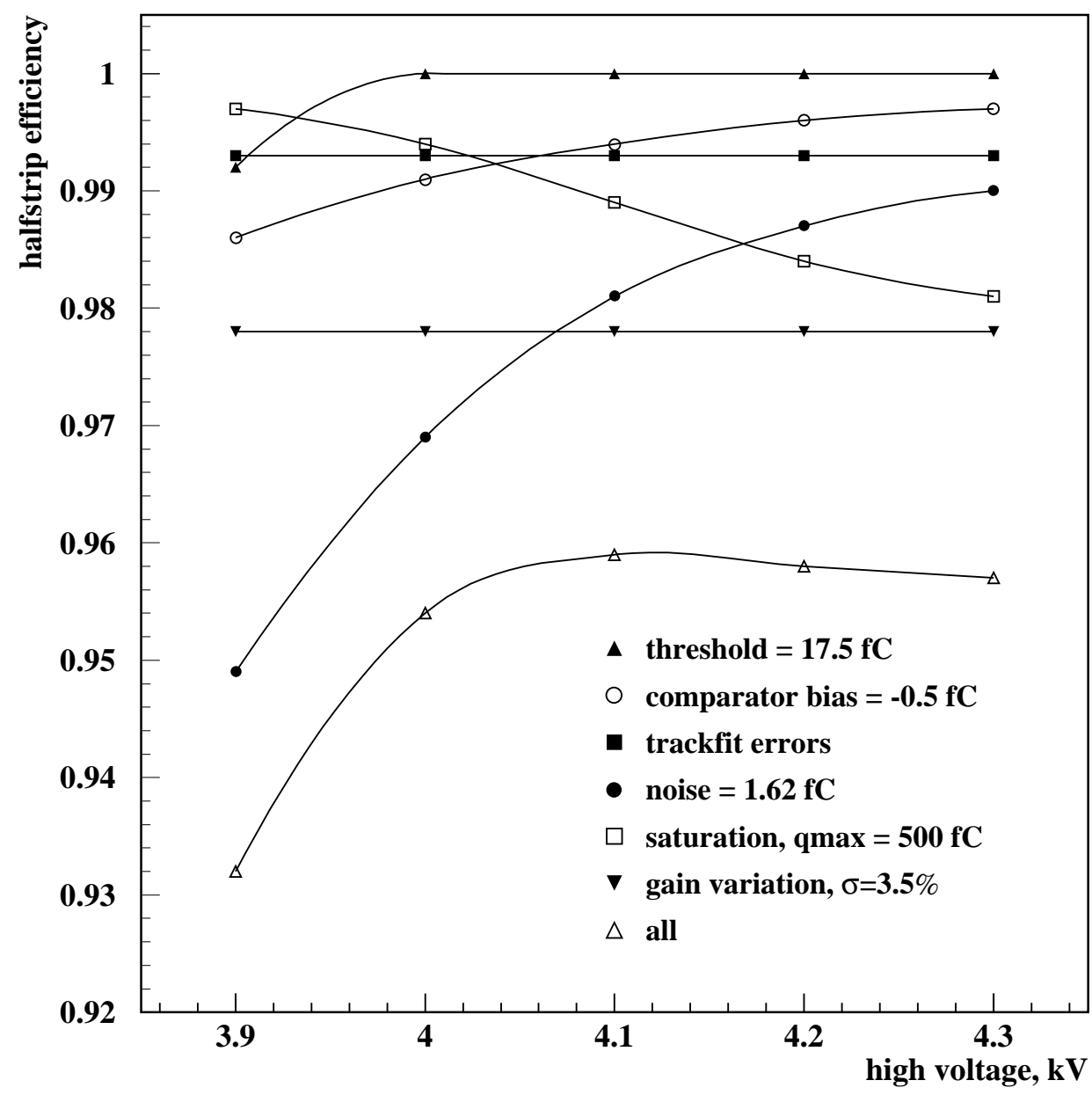

Fig. 9. Monte Carlo trigger halfstrip efficiency as a function of high voltage, with individual and combined error sources.

\section{LCT-finding performance}

Once the software trigger (simulation) was carefully tuned to match the performance of the hardware prototype, the next step was to apply it to all six layers of the chamber, and extend its logic to also include the LCT-finding algorithm which converts the halfstrip patterns into identified local charged tracks. The trigger simulation could then be applied to any of the CSC data to predict what the trigger result would have been if trigger hardware had existed for the whole chamber.

The LCT-finding procedure compares the pattern of halfstrip hits in the six layers with a set of predefined prioritized and numbered patterns called trigger codes. Each code is assigned an angle $\alpha$ and a position $x$ which are the average crossing angle and position (at the center of the chamber) of all perfectly straight tracks which could match the corresponding pattern. The tracks used to compute these averages have flat distributions in $x$ and $\alpha$. The priority of a pattern is determined by $\alpha$, with the highest priority given to perpendicular tracks. A code is required to match a (data) pattern in at least four of the possible six layers, and, if several codes match the same pattern, the rule followed is to choose the highest priority code among 
those which match the maximum possible number of layers. (For example, any 6-layer code is preferred to any 5-layer code.) Only one LCT is returned per event. A complete description of the codes used in this study is given in the appendix.

For the six-layer case, the trigger performance can't be fairly measured by using the same CSC data to find the track, because the track and trigger position measurements are correlated. In addition, it is not always possible to unambiguously find a track. So, the H2 Silicon Beam Telescope (SiBT) located five meters upstream of the CSC was used to identify and locate the track. Since the telescope was upstream of the iron absorber, it could identify the muon track even for muon shower events.

As an example, Fig. 10 shows an event from the data taken with the iron absorber upstream of the chamber.

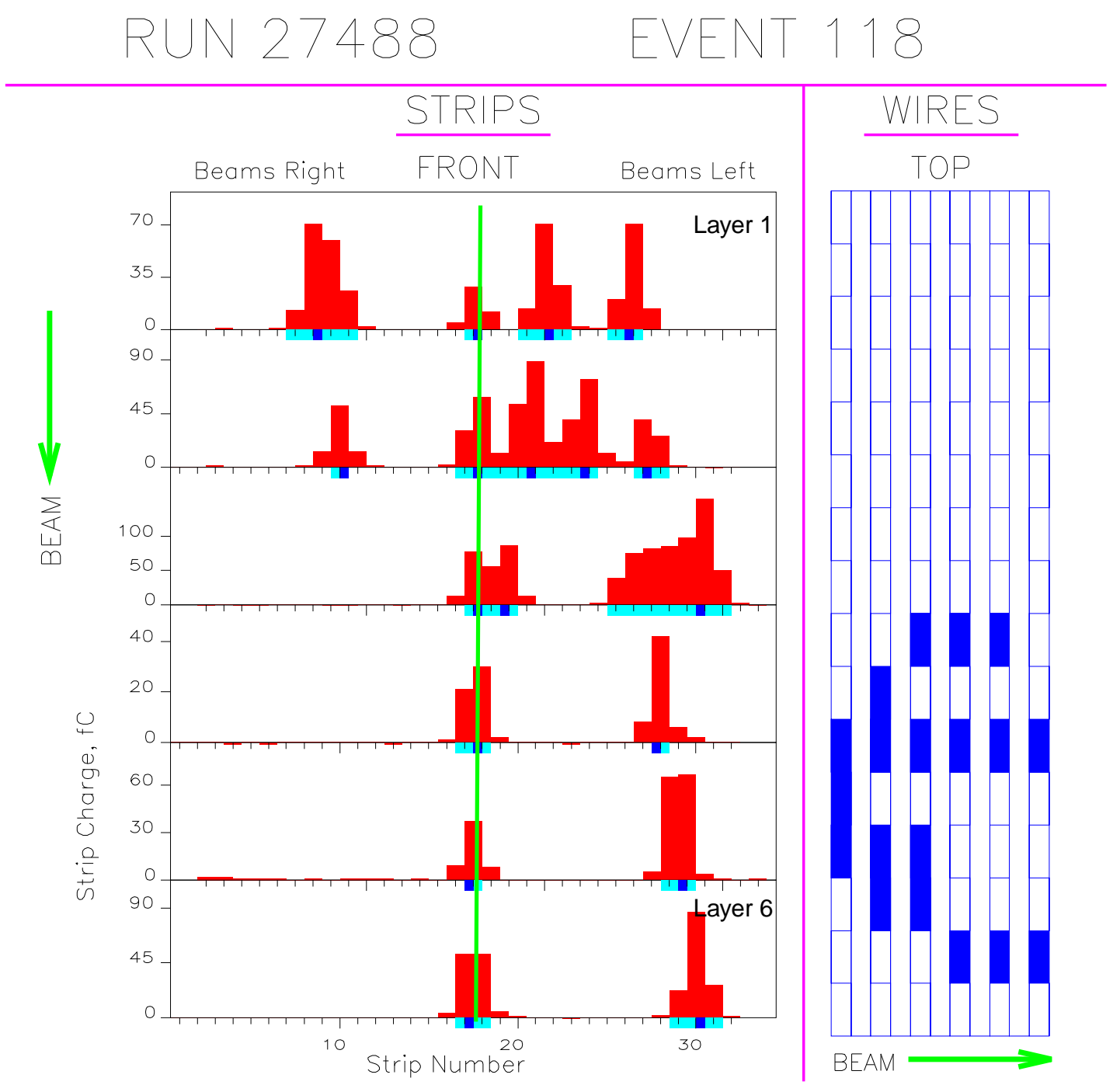

Fig. 10. A sample event. The left side of the plot shows the charge on each of the 32 strips in each of the six layers, while the right side shows the information from groups of anode wires (not used in this study). Strips with charge above the trigger threshold have shading below the axes, while the halfstrip peaks are marked with dark squares. The vertical line at $x=17.56$ shows the track position extrapolated from the silicon telescope. This event was assigned a 6-layer code 7 and track position $x=17.59$.

The event display shows the charge on each strip, the strips-over-threshold and peak halfstrips identified by the software trigger, and the extrapolated track from the SiBT. Although this charge distribution would be 
a complicated problem for the offline track reconstruction software, the pattern of halfstrip peaks is considerably simpler, and the resulting LCT code is a good match to the SiBT track.

The trigger efficiency was defined as the fraction of events for which the track position from the trigger was within a specified distance of the extrapolated track position as measured by the SiBT. No cuts were made on the CSC data, and no cuts on the SiBT data except to require at least one SiBT track in the $x$ projection. (In the fewer than $0.1 \%$ of cases where there was more than one SiBT track in an event, the first one was used.) Figure 11a shows the distribution of $\Delta x=x$ (trigger code) $-x$ (SiBT) for data taken at $4.0 \mathrm{kV}$ (100 fC Landau peak) with a $300 \mathrm{GeV} / \mathrm{c}$ muon beam, and with the $30 \mathrm{~cm}$ iron absorber in place. For $98.9 \%$ of the events, halfstrip LCTs were found. The $x$ measurement error has a standard deviation of 0.11 stripwidths, or $0.7 \mathrm{~mm}$. The corresponding efficiencies are: $95.2 \%$ with $|\Delta x|<0.5$ stripwidths, and $96.5 \%$ with $|\Delta x|<1$ stripwidth. The shaded histogram superimposed in Fig. 11a indicates the subset of 1044 events which did not consist of simple isolated tracks.
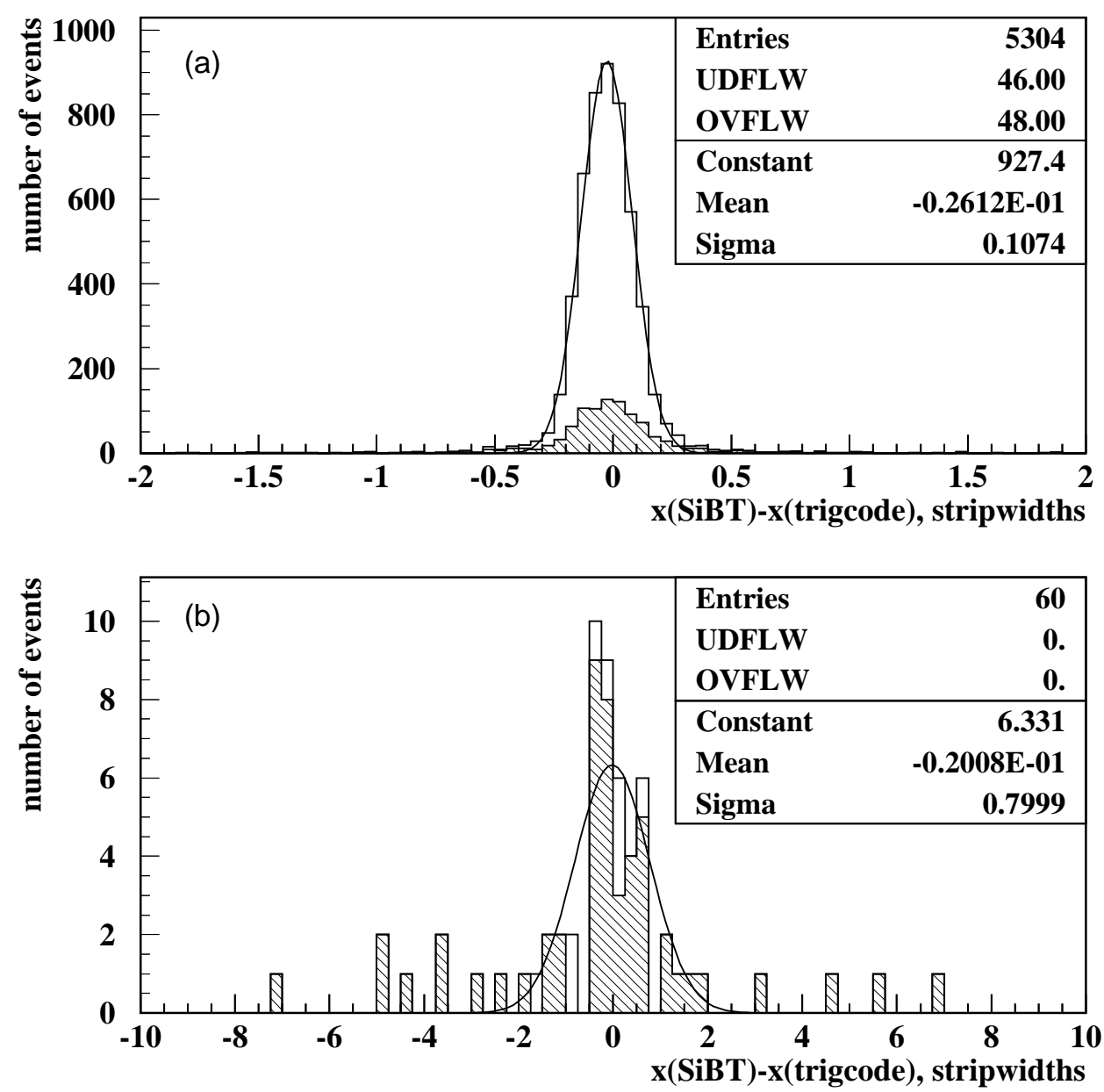

Fig. 11. LCT resolution with comparator halfstrip inputs, for data with $300 \mathrm{GeV} / \mathrm{c}$ muons and $30 \mathrm{~cm}$ of iron in front of the CSC. The residual distributions are shown separately for events with halfstrip codes (a) and events with only double-strip codes (b). Codes were found for all 5364 events. The shaded histograms show the contributions from events which were not simple isolated tracks.

To roughly estimate the probability of muon bremsstrahlung radiation, we used a criterion of the number of planes with multiple charge cluster peaks: events were called "clean" only if no more than one plane had 
multiple cluster peaks. The fraction of clean events was $94 \%$ for data taken without the iron block in the

Tab. 2. LCT-finding probabilities for different event types and spatial accuracies.

\begin{tabular}{l|cccc}
\hline \multicolumn{1}{c|}{ Event Type } & $\begin{array}{c}\text { Event Type } \\
\text { Fraction }\end{array}$ & $\begin{array}{c}|\Delta x|<0.5 \\
\text { Fraction }\end{array}$ & $|\Delta x|<1$ Fraction & $\begin{array}{c}1 / 2 \text {-strip LCT } \\
\text { found }\end{array}$ \\
\hline All & $100 \%$ & $95.2 \%$ & $96.5 \%$ & $98.9 \%$ \\
Clean & $80 \%$ & $98.8 \%$ & $99.4 \%$ & $99.8 \%$ \\
Others & $20 \%$ & $81.0 \%$ & $85.5 \%$ & $95.3 \%$ \\
\hline
\end{tabular}

muon path, and dropped to $80 \%$ for data taken with the iron block. We did not observe a significant difference in these numbers for muons of 150,225 and $300 \mathrm{GeV} / \mathrm{c}$ momenta. Table 2 shows the probabilities of finding LCTs and their spatial localization (in stripwidth units) for the $80 \%$ "clean" and 20\% "other" events separately.

In the muon trigger design, the halfstrip patterns which have just been described are used to find LCTs which have high-precision position information for high-momentum muon tracks. For low-momentum muons, which are more subject to multiple scattering, the precision is not needed. Therefore, since low $\mathrm{p}_{\mathrm{T}}$ tracks have larger bending angles, double-strip patterns with one-fourth the granularity are also generated by the trigger logic for each event, by or-ing four adjacent halfstrip bits. This allows larger-angle tracks to be found without a large increase in the number of predefined trigger codes. Figure $11 \mathrm{~b}$ shows the $\Delta x$ distribution for events where no halfstrip code was satisfied, but a double-strip code was found. Again, the shaded histogram shows the subset of events (51) which were not clean events; it accounts for almost all of them. An example of one of these other events is shown in Fig. 12. The efficiencies for finding either a halfstrip code or a double-strip code are: $95.7 \%$ with $|\Delta x|<0.5$ stripwidths, $97.3 \%$ with $|\Delta x|<1$ stripwidth, and $100 \%$ with no limit on $|\Delta x|$.

\section{Comparison with digital method}

An alternative way to find cluster peaks, called the digital method [6], is to find the centers of groups of adjacent strips which are over threshold. For instance, if strips 1,2 and 3 are above threshold, the peak will be assigned the coordinate of the center of the middle strip, i.e. 2. If only the two strips 2 and 3 are above threshold, the peak coordinate will be 2.5 . Thus, this method also provides hit localization with halfstrip steps; however, its performance is less stable.

The decision returned by this scheme depends on the actual threshold or signal amplitude. This is obvious from considering the charge cluster of the three strips shown in Fig. 1. By raising the threshold from very low to a higher and higher value, one can see that the answer changes from 2 to 2.5 as the left strip charge sinks under the threshold, and then swings back to 2 as the threshold goes over the right strip charge. Varying the total charge in a cluster results in a similar effect.

Also, for a given hit position relative to the strips, the answer provided by the digital scheme is clearly stripwidth dependent. Wide strips tend to have clusters with a small number of strips over threshold, while narrow strips have clusters with a larger number of strips affected. Thus, if the strip width changes in a chamber, it creates additional uncertainties.

And last, probably the most serious disadvantage of the scheme is its higher vulnerability to backgrounds: (a) overlapping clusters are not resolved at all and (b) even small side tails may cause a dramatic shift in the answer.

To have a direct comparison of the two schemes (comparators vs. digital), we used the same data as 


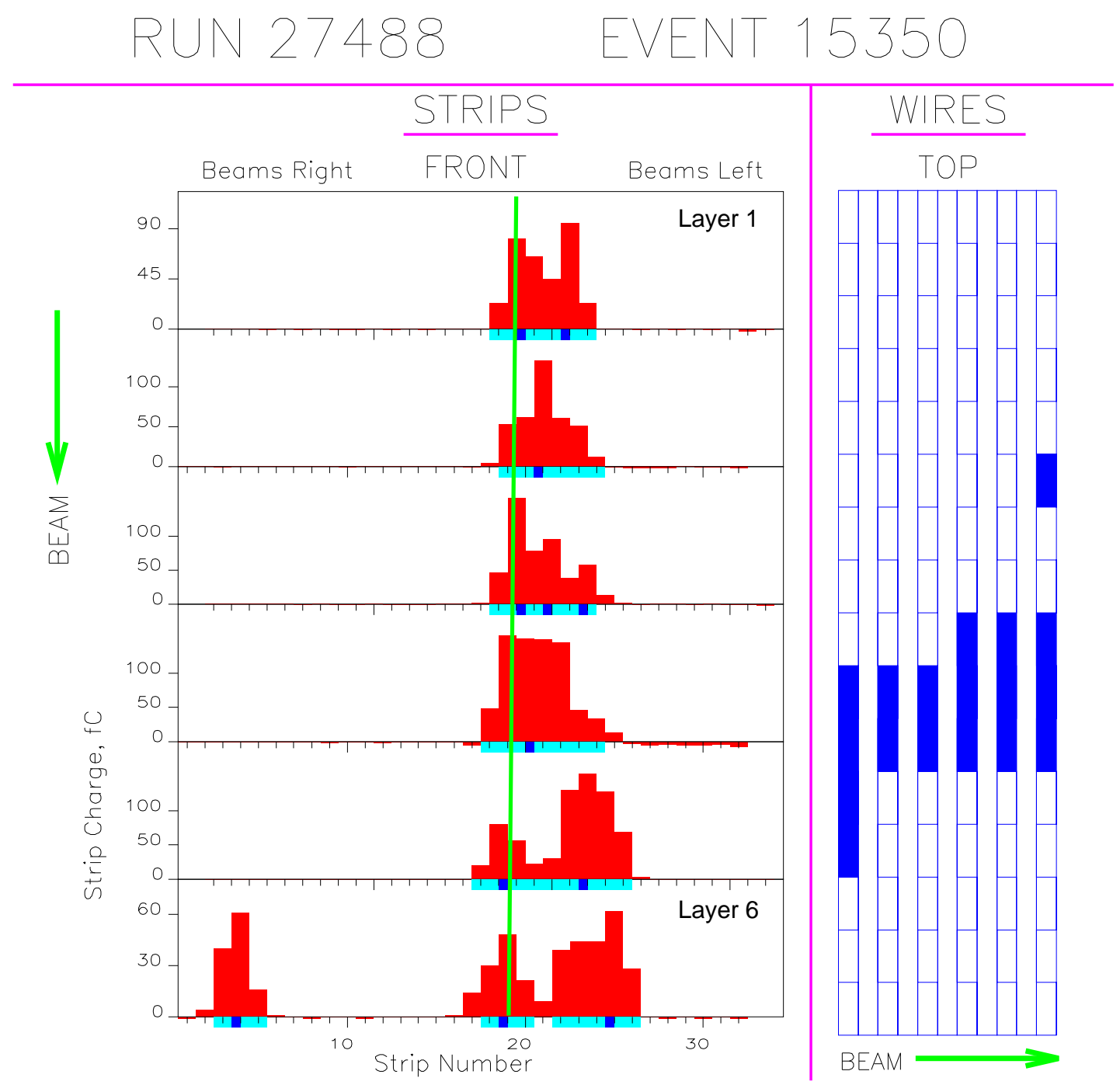

Fig. 12. An event with no halfstrip code, but with double-strip code $7, x=19.36$. The silicon telescope extrapolated track (vertical line) was at $x=19.24$. (The incorrect halfstrip peak in layer 4 is a consequence of the random errors introduced in the software trigger as described in Sect. 2.2.

described in Sect. 4 to simulate the digital scheme performance. Figure 13 shows this algorithm applied to the same event that was shown in Fig. 10. The single-layer halfstrip and one-strip efficiencies, defined as in Sect. 3.1, were measured using the digital method and the over-threshold bits from the comparator prototype. They were found to be $68 \%$ for $|\Delta x|<0.5$ and $96 \%$ for $|\Delta x|<1$, showing that although the digital method locates peaks with halfstrip granularity, it has effectively one-strip resolution. Since the performance of the digital method is sensitive to the value of the threshold, these efficiencies were also evaluated by software simulation for a variety of other thresholds. This check showed that by chance the maximum efficiencies are reached just at the $15 \mathrm{fC}$ threshold of the comparator prototype.

The LCT-finding simulation was then repeated using digital halfstrips (with threshold $15 \mathrm{fC}$ ) as inputs instead of comparator halfstrips. The results, comparable to Fig. 11, are shown in Fig. 14. The halfstrip efficiencies are: $89.1 \%$ with $|\Delta x|<0.5$ stripwidths, $91.8 \%$ with $|\Delta x|<1$ stripwidth, and $93.9 \%$ with no limit on $|\Delta x|$, and the half or double-strip efficiencies are $91.3 \%$ with $|\Delta x|<0.5$ stripwidths, $95.2 \%$ with $|\Delta x|<1$ stripwidth, and $99.6 \%$ with no limit on $|\Delta x|$. The performance of the digital algorithm probably could be improved by expanding the set of allowed trigger codes to take into account its intrinsic one-strip resolution (for example, by recognizing patterns with one or two misplaced halfstrips). However, this 


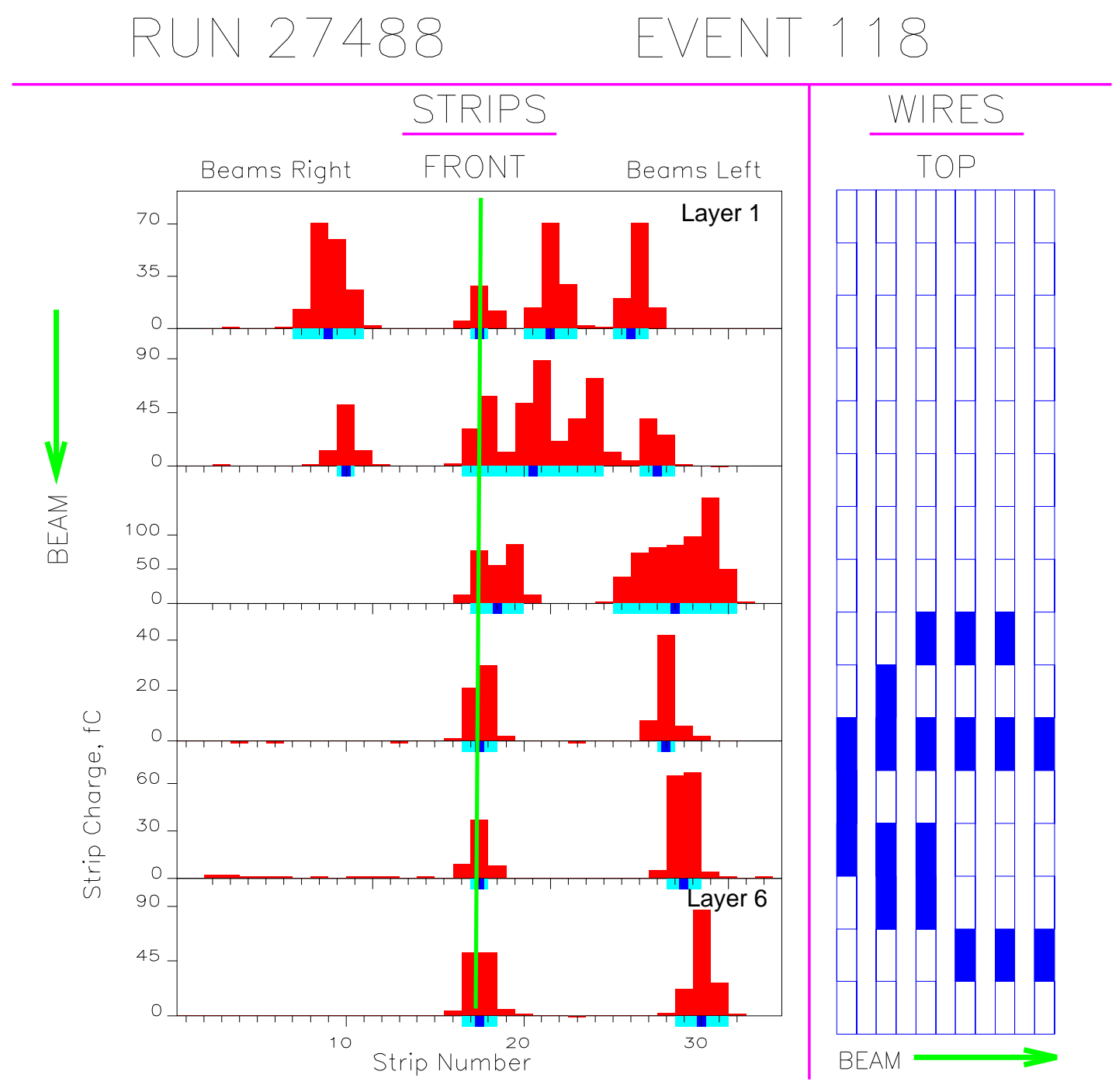

Fig. 13. The same event as in Fig. 10, except here the digital method (with $15 \mathrm{fC}$ threshold) has been used to locate the peak halfstrips. Note that the possible peak positions are shifted by $1 / 4$ strip. This event was assigned a 4-layer code 1, with layers 2 and 3 missing, and with $x=17.25$.

worse resolution makes it unlikely that the digital method can ever match the performance of the comparators even for simple events.

\section{Conclusion}

A prototype comparator network board was built and tested in a high-energy muon beam. Muon hit localization in a chamber plane within a halfstrip wide bucket with $92 \%$ probability was obtained. Based on this performance and data obtained in the beam, we showed that patterns of "halfstrip" hits in the six-plane chamber allowed us to identify high-energy muons in the presence of bremsstrahlung radiation with about $99 \%$ probability and $0.7 \mathrm{~mm}$ spatial precision for $6.35 \mathrm{~mm}$ wide strips. 

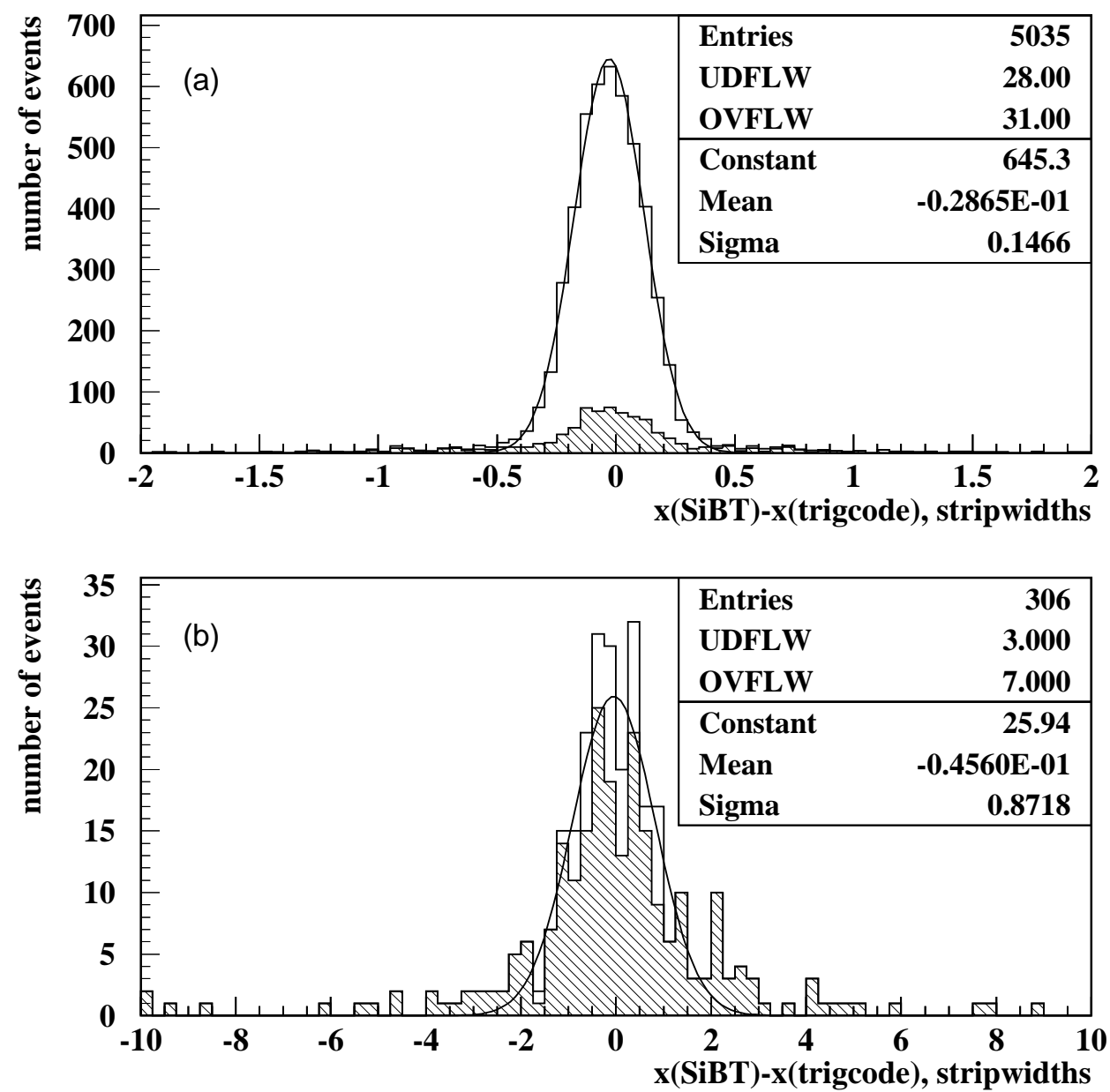

Fig. 14. LCT resolution results when halfstrips are identified by the digital method for data with an iron absorber in front of the CSC: a) events with halfstrip codes, b) events with only double-strip codes. There were 23 events with no code. The shaded histograms show the contributions from events which were not simple isolated tracks.

\section{Appendix: LCT codes}

The trigger patterns used by the software were defined using a simplified model of the chamber geometry: $6.35 \mathrm{~mm}$ strip pitch, the measured layer spacing (approximately $26.6 \mathrm{~mm}$ ), and perfect layer-to-layer alignment. A set of straight lines was generated with $0<x<0.5$ stripwidths, $|\alpha|<100 \mathrm{mrad}$, and flat distributions in $x$ and $\alpha$. All patterns which could be made by these lines crossing the chamber geometry were recorded, along with their average $x$ and $\alpha$. The 41 patterns with the smallest average $\alpha$ were kept as LCT codes; they include all patterns up to four halfstrips wide, and some with five. The distribution of these codes in $x$ - $\alpha$ space is shown in Fig. 15. The patterns are described in Table 3 by giving the offsets of the halfstrips in each layer relative to the highest-numbered halfstrip in the pattern. The last column labeled $x$ gives the $x$ position assigned to the pattern (derived from the average $x$ ), where $x$ is measured in stripwidths and is the offset to be added to the coordinate of the highest-numbered halfstrip, which is defined to be the strip number minus 0.5 for left halfstrips, and the strip number for right halfstrips. 
To completely define a set of codes, some arbitrary assignments of priority had to be made among patterns which are equivalent except for reflections. The rule followed is that patterns which are left-right reflections are always given adjacent priorities, with codes with $\langle\alpha\rangle\langle 0$ receiving the higher priority. If the best code / pattern match occurs at more than one place in the same event, the pattern with the highest strip coordinate is selected.

Tab. 3. LCT code definitions.

\begin{tabular}{|c|c|c|c|c|c|c|c|c|c|}
\hline code & $\begin{array}{l}\text { pattern width } \\
\text { in halfstrips }\end{array}$ & L1 & L2 & L3 & L4 & L5 & L6 & $x$, stripwidths & $\langle\alpha\rangle$, radians \\
\hline 1 & 1 & 0 & 0 & 0 & 0 & 0 & 0 & .250 & 0 \\
\hline 2 & 2 & -1 & -1 & -1 & -1 & -1 & 0 & -.147 & -.018 \\
\hline 3 & 2 & 0 & 0 & 0 & 0 & 0 & -1 & .147 & .018 \\
\hline 4 & 2 & -1 & 0 & 0 & 0 & 0 & 0 & .147 & -.018 \\
\hline 5 & 2 & 0 & -1 & -1 & -1 & -1 & -1 & -.147 & .018 \\
\hline 6 & 2 & -1 & -1 & -1 & -1 & 0 & 0 & -.090 & -.023 \\
\hline 7 & 2 & 0 & 0 & 0 & 0 & -1 & -1 & .090 & .023 \\
\hline 8 & 2 & -1 & -1 & 0 & 0 & 0 & 0 & .090 & -.023 \\
\hline 9 & 2 & 0 & 0 & -1 & -1 & -1 & -1 & -.090 & .023 \\
\hline 10 & 2 & -1 & -1 & -1 & 0 & 0 & 0 & .000 & -.029 \\
\hline 11 & 2 & 0 & 0 & 0 & -1 & -1 & -1 & .000 & .029 \\
\hline 12 & 3 & -2 & -1 & -1 & -1 & -1 & 0 & -.250 & -.031 \\
\hline 13 & 3 & 0 & -1 & -1 & -1 & -1 & -2 & -.250 & .031 \\
\hline 14 & 3 & -2 & -1 & -1 & -1 & 0 & 0 & -.157 & -.043 \\
\hline 15 & 3 & 0 & -1 & -1 & -1 & -2 & -2 & -.343 & .043 \\
\hline 16 & 3 & -2 & -2 & -1 & -1 & -1 & 0 & -.343 & -.043 \\
\hline 17 & 3 & 0 & 0 & -1 & -1 & -1 & -2 & -.157 & .043 \\
\hline 18 & 3 & -2 & -1 & -1 & 0 & 0 & 0 & -.066 & -.049 \\
\hline 19 & 3 & 0 & -1 & -1 & -2 & -2 & -2 & -.434 & .049 \\
\hline 20 & 3 & -2 & -2 & -2 & -1 & -1 & 0 & -.434 & -.049 \\
\hline 21 & 3 & 0 & 0 & 0 & -1 & -1 & -2 & -.066 & .049 \\
\hline 22 & 3 & -2 & -2 & -1 & -1 & 0 & $\overline{0}$ & -.250 & -.056 \\
\hline 23 & 3 & 0 & 0 & -1 & -1 & -2 & -2 & -.250 & .056 \\
\hline 24 & 4 & -3 & -2 & -2 & -1 & -1 & 0 & -.500 & -.061 \\
\hline 25 & 4 & 0 & -1 & -1 & -2 & -2 & -3 & -.500 & .061 \\
\hline 26 & 4 & -3 & -3 & -2 & -2 & -1 & 0 & -.676 & -.070 \\
\hline 27 & 4 & 0 & 0 & -1 & -1 & -2 & -3 & -.324 & .070 \\
\hline 28 & 4 & -3 & -2 & -1 & -1 & 0 & 0 & -.324 & -.070 \\
\hline 29 & 4 & 0 & -1 & -2 & -2 & -3 & -3 & -.676 & .070 \\
\hline 30 & 4 & -3 & -3 & -2 & -1 & -1 & 0 & -.588 & -.075 \\
\hline 31 & 4 & 0 & 0 & -1 & -2 & -2 & -3 & -.412 & .075 \\
\hline 32 & 4 & -3 & -2 & -2 & -1 & 0 & 0 & -.412 & -.075 \\
\hline 33 & 4 & 0 & -1 & -1 & -2 & -3 & -3 & -.588 & .075 \\
\hline 34 & 4 & -3 & -3 & -2 & -1 & 0 & 0 & -.500 & -.087 \\
\hline 35 & 4 & 0 & 0 & -1 & -2 & -3 & -3 & -.500 & .087 \\
\hline 36 & 5 & -4 & -3 & -2 & -2 & -1 & 0 & -.750 & -.091 \\
\hline 37 & 5 & 0 & -1 & -2 & -2 & -3 & -4 & -.750 & .091 \\
\hline 38 & 5 & -4 & -3 & -3 & -2 & -1 & 0 & -.856 & -.093 \\
\hline 39 & 5 & 0 & -1 & -1 & -2 & -3 & -4 & -.644 & .093 \\
\hline 40 & 5 & -4 & -3 & -2 & -1 & -1 & 0 & -.644 & -.093 \\
\hline 41 & 5 & 0 & -1 & -2 & -3 & -3 & -4 & -.856 & .093 \\
\hline
\end{tabular}

The number of patterns actually present in the data depends on the mean value and range of the track angle 


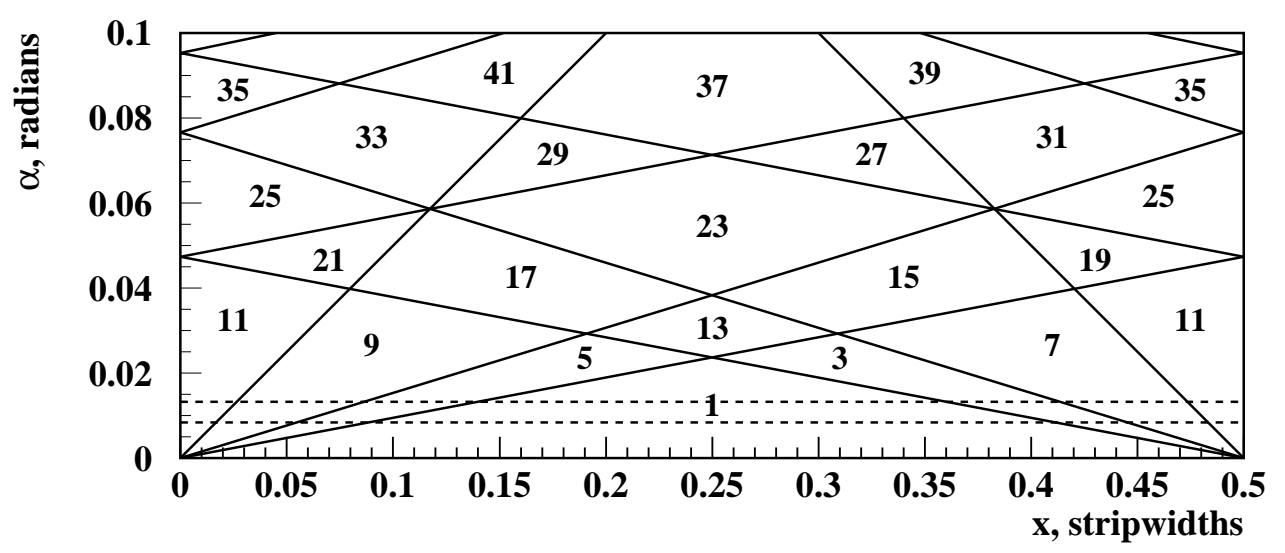

Fig. 15. The partitioning of $x-\alpha$ parameter space among the defined trigger codes. Only codes with $\alpha>0$ are shown. The dashed lines show the $2 \sigma$ range of angles in the CSC data: $10.8 \pm 2.4 \mathrm{mrad}$.

$\alpha$. This can be seen from Fig. 15, where the dashed lines show the $2 \sigma$ range of angles in our beam data; only the codes $1,3,5,7,9$ and 11 occur with any significant frequency. Since the $x$ resolution of individual patterns varies (some codes pinpoint the track quite precisely, while others are not that good), the overall spatial resolution therefore also depends on $\langle\alpha\rangle$ and $\Delta \alpha$, as shown for some examples in Fig. 16.

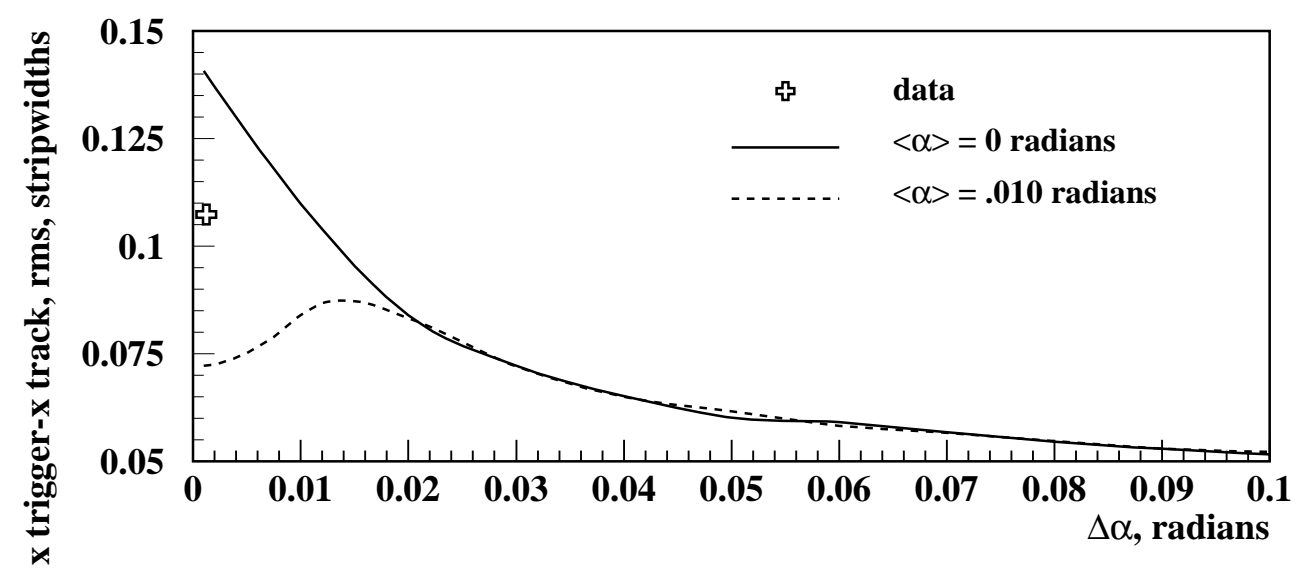

Fig. 16. The dependence of the trigger spatial resolution on the range of track angles. The curves are calculated purely from geometry, with $\alpha$ uniformly distributed in the range $\langle\alpha\rangle \pm \Delta \alpha$.

\section{References}

[1] CMS Muon Technical Design Report, CERN/LHC 97-32, 1997.

[2] J. Hauser, "Baseline Design for CSC based Endcap Muon Trigger", CMS TN/95-013.

[3] A. Korytov, "Analysis of valid hit patterns in Cathode Strip Chambers", CMS TN/94-212.

[4] M. Baarmand et al., Nucl. Instr. and Meth. A 402 (1998) 36.

[5] J.C. Santiard et al., "GASPLEX: A low noise analog signal processor for readout of gaseous detectors", CERN-ECP/94-17.

[6] M. Gorski et al., "Clusters in RPC and Muon Trigger Performance”, CMS TN/95-107. 